(C) [2008] IEEE. Reprinted, with permission, from [Steven W. Su, Hung Nguyen and Q. P. Ha, I ntegral Controller Design for Nonlinear Systems Using Inverse Optimal Control, Control, Automation, Robotics and Vision, 2008. ICARCV 2008. 10th International Conference on, 17-20 Dec. 2008]. This material is posted here with permission of the IEEE. Such ermission of the IEEE does not in any way imply IEEE endorsement of any of the University of Technology, Sydney's products or services. Internal or personal use of this material is permitted. However, permission to reprint/republish this material for advertising or promotional purposes or for creating new collective works for resale or redistribution must be obtained from the IEEE by writing to pubs-permissions@ieee.org. By choosing to view this document, you agree to all provisions of the copyright laws protecting it 


\title{
I ntegral Controller Design for Nonlinear Systems Using Inverse Optimal Control
}

\author{
Steven W. Su†, Hung Nguyen $\dagger$, and Q. P. Ha† \\ $\dagger$ Faculty of Engineering and Information Technology \\ University of Technology, Sydney \\ Broadway NSW 2007 Australia \\ (Steven.Su, Hung.Nguyen, Quang.Ha)@uts.edu.au
}

\begin{abstract}
This paper proposes an integral controller design scheme for nonlinear systems based on optimal control and the passivity theorem in order to suppress the effect of external disturbances. The main strategy is to augment an optimal controller with a PI type controller. To guarantee the proposed controller has a desired stability margin, the passivity-based design method is introduced. Here, the inverse optimal control technique is employed to avoid the need of solving a HamiltonJacobi equation. An illustrative example is given to show the design procedure and the controller effectiveness.
\end{abstract}

\section{INTRODUCTION}

An important objective of control system design is to minimise the effects of external disturbances. The problem of disturbance rejection arises in many industrial fields, such as motion-control, active noise control and vibration control [1].

In order to cope with the problem of constant disturbance rejection for nonlinear systems, nonlinear $\mathcal{H}_{\infty}$ methods [3], [4] have been used, see for example, [2]. That paper extended the concept of comprehensive stability for linear systems [5], [6] to deal with the nonlinear disturbance suppression problem. The main bottleneck for nonlinear state feedback $\mathcal{H}_{\infty}$ control, which is similar to the problem encountered in nonlinear optimal control, is the need to solve a Hamilton-Jacobi (HJ) partial differential equation (PDE) [7]. Although the method proposed in [2] can reduce the order of the HJ PDE involved, solving a HJ PDE in general is not feasible.

In [1], a relatively practical approach, based on singular perturbation analysis [8], directly adds an integrator to an already existing controller to achieve constant disturbance rejection, while still retaining the stability of the system. However, [1] showed only the existence of such integrator augmented controller but did not consider robustness of the system with model uncertainties. In this paper, we investigate the design of a robust nonlinear integral controller based on the passivity theory [9], [10], [11] and inverse optimal control strategies [10], [12]. Here, an effective method will be proposed which can result in a desired stability margin without the need of solving a HJ PDE.

The remainder of the paper is organised as follows. Following Section II providing a background on passivity and small gain theorems, Section III summarises results on the relationship between passivity and stability margins of optimal nonlinear systems. In Section IV, we will give the main results of this paper. Section V presents our discussion and Section VI provides an illustrative example. Finally, a conclusion is withdrawn in Section VII.

\section{PREMilinary}

In this section, some required knowledge and background, such as small gain and passivity theorems will be introduced.

\section{A. Small gain and passivity theorems}

The small gain theorem is probably one of the most commonly used tools for stability analysis of feedback control structures.

$\begin{array}{lllll}\mathrm{r} & \mathrm{u}_{1} & \mathrm{H}_{1} & \mathrm{y}_{1} & \mathrm{y}\end{array}$

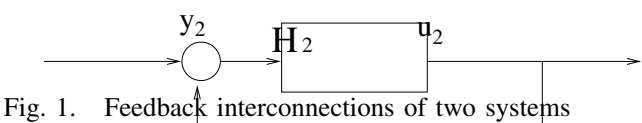

Theorem 1: (Small gain theorem) [13]:

Consider a closed-loop control system $\Sigma^{f} H_{1} H_{2}$ shown in Figure 1. Suppose that both $H_{1}$ and $H_{2}$ have finite $\mathcal{L}_{q}$ gains given respectively as $\gamma_{q}\left(H_{1}\right)$ and $\gamma_{q}\left(H_{2}\right)(q \in$ $\{1,2, \ldots, \infty\})$. Then the closed-loop system $\Sigma^{f} H_{1} H_{2}$ is $\mathcal{L}_{q^{-}}$ stable if $\gamma_{q}\left(H_{1}\right) \gamma_{q}\left(H_{2}\right)<1$.

Proof: See Page 16-17 in [13].

Definition 1: (Dissipativity) [10]:

Assume that associated with the system $H$ is a function $w: \mathbb{R}^{m} \times \mathbb{R}^{m} \mapsto \mathbb{R}$, called the supply rate, which is locally integrable for every $u \in U$, that is, it satisfies $\int_{t_{0}}^{t_{1}} \mid w\left(u(t), y(t) \mid d t<\infty\right.$ for all $t_{0} \leq t_{1}$. Let $X$ be a connected subset of $\mathbb{R}^{n}$ containing the origin. We say that the system $H$ is dissipative in $X$ with respect to the supply rate $w(u, y)$ if there exists a function $S(x) \geq 0, S(0)=0$, such that for all $x \in X$,

$$
S(x(T))-S(x(0)) \leq \int_{0}^{T} w(u(t), y(t)) d t
$$

for all $u \in U$ and all $T \geq 0$, such that $x(t) \in X, \forall t \in[0, T]$. The function $S(x)$ is then called a storage function.

Definition 2: (Passivity)[101 
System $H$ is said to be passive if it is dissipative with supply rate $w(u, y)=u^{T} y$.

Theorem 2: (Feedback interconnections of passive systems) [10]:

Suppose that $H_{1}$ and $H_{2}$ are passive. Then the closed loop system $\Sigma^{f} H_{1} H_{2}$ shown in Figure 1 is also passive.

Proof: See Page 33 in [10].

Definition 3: (Excess/Shortage of Passivity) [10]:

System $H$ is said to be

- Output Feedback Passive (OFP) if it is dissipative with respect to $w(u, y)=u^{T} y-\rho y^{T} y$ for some $\rho \in \mathbb{R}$.

- Input Feedforward Passive (IFP) if it is dissipative with respect to $w(u, y)=u^{T} y-\nu u^{T} u$ for some $\nu \in \mathbb{R}$.

In order to deal with the internal stability issue, let us review the definitions of detectability and observability. [10]:

Definition 4: (Zero-state detectability and observability)

Consider the system $H$ with zero input, that is $\dot{x}=f(x, 0)$, $y=h(x, 0)$, and let $Z \subset \mathbb{R}^{n}$ be its largest positively invariant set contained in $\left\{x \in \mathbb{R}^{n} \mid y=h(x, 0)=0\right\}$. We say that $H$ is zero-state detectable (ZSD) if $x=0$ is asymptotically stable conditionally to $Z$. If $Z=\{0\}$, we say that $H$ is zero-state observable (ZSO).

Definition 5: (Zero-input detectability) [10]:

The system $H$ is said to be zero-input detectable (ZID) if $y \equiv 0$ implies $u(t) \rightarrow 0$ as $t \rightarrow \infty$.

Theorem 3: (feedback interconnections of OFP/IFP systems) [10]:

If $H_{1}$ and $H_{2}$ are dissipative with radially unbounded storage functions $S_{1}$ and $S_{2}$ then the equilibrium $(0,0)$ of their feedback interconnection is:

(i) Global Stability (GS), if $H_{1}$ and $H_{2}$ are passive.

(ii) Global Asymptotic Stability (GAS), if $H_{1}$ is GS and $\operatorname{IFP}(\nu)$, and the system $H_{2}$ is ZSD and $\operatorname{OFP}(\rho)$ with $\nu+\rho>0$.

Proof: See Page 52-53 in [10].

For linear systems, there are two important concepts in the frequency domain, namely gain and phase. Based on these two concepts, the frequency domain tools are developed. For nonlinear systems, $\mathcal{L}_{2}$-gain can be regarded as a generalization of a linear gain while passivity is associated with a phase.

\section{B. Stability margin}

As dynamic systems are usually subject to modelling uncertainties and external disturbances, we need to design a robust controller with some required stability margin. For linear systems, gain margin and phase margin are two essential criteria. In this subsection, we mainly consider a nonlinear disk margin as a kind of structure stability margin for nonlinear systems, which can tolerate input uncertainties.

Consider a nonlinear feedback system shown in Figure 2, where $u$ and $y$ are of the same dimension, and $\Delta$ represents modelling uncertainty. In the nominal case, $\Delta$ is identity and the feedback loop consists of the (nominal) nonlinear plant
$H$ with the nominal control $u=-k(x)=:-y$. We denote the nominal system by $(H, k)$ and the perturbed system by $(H, k, \Delta)$.

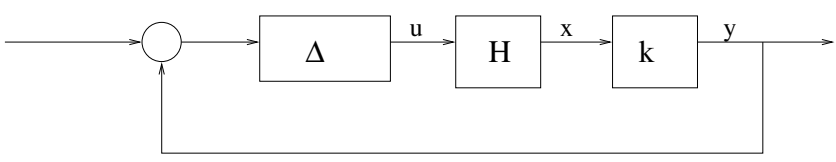

Fig. 2. Nonlinear feedback loop with control law $k(x)$ and input uncertainty $\Delta$.

The block diagram shown in Figure 2 restricts modelling uncertainties to be at the input. This is a common physical situation, in particular when simplified models of actuators are used for the design. Here, we assume a relative degree of 0 for the input disturbance uncertainty $\Delta$ while the input disturbance uncertainty may be static or dynamic.

Definition 6: (Disk margin) [10]:

The nonlinear feedback system $(H, k)$ is said to have a disk margin $D(\alpha)$ if the closed-loop system $(H, k, \Delta)$ is GAS for any $\Delta$ which is $\operatorname{GAS}$ and $\operatorname{IFP}(\nu), \nu>\alpha$, with a radially unbounded storage function.

\section{PASSIVITY AND STABILITY MARGINS OF OPTIMAL SYSTEMS}

In this section, we briefly review the relationship between passivity and optimality for nonlinear systems.

Theorem 4: (Optimality and passivity) [10]:

The law $u=-k(x)$ is an optimal stabilizing control for the system shown in Figure 2, in a special case where $R(x)=I$ in the following cost function:

$$
J=\int_{0}^{\infty}\left(l(x)+u^{T} R(x) u\right) d t,
$$

iff the system

$$
\left\{\begin{array}{l}
\dot{x}=f(x)+g(x) u \\
y=k(x)
\end{array}\right.
$$

is ZSD and $\operatorname{OFP}\left(-\frac{1}{2}\right)$ with a $\mathcal{C}^{1}$-storage function $S(x)$.

Proof: See Page 95-96 in [10].

Theorem 5: (Disk margin of optimal stabilization) [10]:

If the law $u=-k(x)$ is an optimal globally stabilizing control in the special case $R(x)=I$ for the cost function (1), then $u=-k(x)$ achieves a disk margin $D\left(\frac{1}{2}\right)$.

Proof: This is a direct result of Theorem 4 .

Based on the relationship between optimality and stability margin, we know that an optimal stabilizing feedback law for the cost function (1) with $R(x)=I$ can be utilised to achieve a disk margin. However, when $R(x) \neq I$, the disk margin is generally not ensured.

Theorem 6: (Scaling and high gain) [10]:

If the law $u=-k(x)$ is an optimal globally stabilizing control in the special case $R(x)=I$ for the cost function (1), then $u=-\frac{1}{\epsilon} k(x), \epsilon \leq 1$, achieves a disk margin $D\left(\frac{\epsilon}{2}\right)$. 


\section{MAIN RESULTS}

We are now ready to present the main results of this paper.

Theorem 7: Suppose system $H_{1}$ is $\operatorname{OFP}(\alpha)$ and $H_{2}$ is $\operatorname{IFP}(\beta)$, then the system of feedback connection as shown in Figure 1 is $\operatorname{OFP}(\alpha+\beta)$.

Proof: As systems $H_{1}$ and $H_{2}$ are $\operatorname{OFP}(\alpha)$ and $\operatorname{IFP}(\beta)$ respectively, there exist two storage functions $S_{1}\left(x_{1}\right)$ and $S_{2}\left(x_{2}\right)$ such that

$$
\begin{aligned}
& \dot{S}_{1}\left(x_{1}\right) \leq u_{1}^{T} y_{1}-\alpha y_{1}^{T} y_{1}, \\
& \dot{S}_{2}\left(x_{2}\right) \leq u_{2}^{T} y_{2}-\beta u_{2}^{T} u_{2} .
\end{aligned}
$$

Now, we construct a storage function for the connected system as

$$
S\left(x_{1}, x_{2}\right)=S\left(x_{1}\right)+S\left(x_{2}\right) .
$$

Then, according the feedback connection, we have

$$
\begin{aligned}
\dot{S}\left(x_{1}, x_{2}\right) & =\dot{S}\left(x_{1}\right)+\dot{S}\left(x_{2}\right) \\
& \leq-(\alpha+\beta) y^{T} y+r^{T} y_{1}-y_{2}^{T} y_{1}+y_{1}^{T} y_{2} \\
& =r^{T} y-(\alpha+\beta) y^{T} y .
\end{aligned}
$$

Q.E.D.

Theorem 8: Suppose system $H_{1}$ is $\operatorname{OFP}(\alpha)$ and $H_{2}$ is $\operatorname{IFP}(\beta)$. Furthermore assume that their respective storage functions $S_{1}\left(x_{1}\right)$ and $S_{2}\left(x_{2}\right)$ are $\mathcal{C}^{1}$. If, in addition, $H_{1}$ is ZID (see Definition 5) and GAS when $u_{1}=0$, and $H_{2}$ is ZSD (see Definition 4), then the system of feedback connection as shown in Figure 1 is asymptotically stable at the equilibrium $\left(x_{1}, x_{2}\right)=(0,0)$ if $\alpha+\beta \geq 0$. Moreover, if $S_{1}\left(x_{1}\right)$ and $S_{2}\left(x_{2}\right)$ are radially unbounded, the asymptotic stability is global.

Proof: As systems $H_{1}$ and $H_{2}$ are $\operatorname{OFP}(\alpha)$ and $\operatorname{IFP}(\beta)$ respectively, there exist two storage functions $S_{1}\left(x_{1}\right)$ and $S_{2}\left(x_{2}\right)$ such that

$$
\begin{aligned}
& \dot{S}_{1}\left(x_{1}\right) \leq u_{1}^{T} y_{1}-\alpha y_{1}^{T} y_{1}, \\
& \dot{S}_{2}\left(x_{2}\right) \leq u_{2}^{T} y_{2}-\beta u_{2}^{T} u_{2} .
\end{aligned}
$$

Now, we construct a storage function for the connected system as

$$
S\left(x_{1}, x_{2}\right)=S\left(x_{1}\right)+S\left(x_{2}\right) .
$$

Using the interconnection identities $u_{1}=-y_{2}$ and $u_{2}=y_{1}$, we have

$$
\begin{aligned}
\dot{S}\left(x_{1}, x_{2}\right) & =\dot{S}\left(x_{1}\right)+\dot{S}\left(x_{2}\right) \\
& \leq-(\alpha+\beta) y^{T} y .
\end{aligned}
$$

Then, all bounded solutions converge to

$$
E=\left\{\left(x_{1}, x_{2}\right) \mid y_{1}=u_{2}=0 .\right\}
$$

As $H_{1}$ is ZID and $y_{1}=y=0$ in $E$, this implies $u_{1}(t) \rightarrow 0$ $\left(y_{2} \rightarrow 0\right)$ as $t \rightarrow \infty$. $H_{1}$ is GAS with $u_{1}=0$, then $x_{1}(t) \rightarrow 0$.

As $y_{2} \rightarrow 0, u_{2} \rightarrow 0$ and $H_{2}$ is $\mathrm{ZSD}, x_{2}(t)$ should converge to zero. Q.E.D.
Now, we propose the augmentation of an integral controller into the optimal stabilizing controller to deal with external disturbances, as shown in Figure 3. Before presenting the main result about design of this integral controller, we introduce the following lemma.

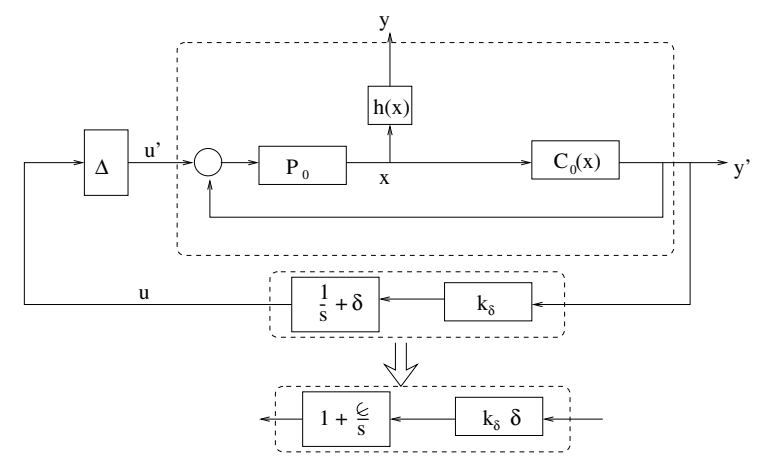

Fig. 3. The proposed control scheme

Lemma 1: The system $H(s)=\delta+\frac{1}{s}$ is $\operatorname{IFP}(\delta)$, and $k H(s)$ is $\operatorname{IFP}(k \delta)$.

Proof: See page 36 and 38 of [10].

Theorem 9: Consider the closed-loop system shown in Figure 3, where it is assumed that the SISO nonlinear system $P_{0}$ has the following form:

$$
P_{0}\left\{\begin{array}{l}
\dot{x}=f(x)+g(x) u \\
y=h(x),
\end{array}\right.
$$

and the system from input $u$ to output $y^{\prime}\left(C_{0} P_{0}\right)$ is of the following form:

$$
C_{0} P_{0}\left\{\begin{array}{l}
\dot{x}=f(x)+g(x) u \\
y^{\prime}=k(x) .
\end{array}\right.
$$

Suppose that $u=-k(x)$ is the optimal stabilizing control law in the special case $R(x)=I$ for the cost function (1). Furthermore, it is assumed that system (7) is ZID. If $\delta$ is chosen to satisfy $\delta>\frac{1}{2 k_{\delta}}$, then the closed-loop system is asymptotically stable at the equilibrium 0 .

Proof: We treat system (7) as $H_{1}$ in Theorem 7 and 8, and $k_{\delta}\left(\frac{1}{s}+\delta\right)$ as $H_{2}$. According to Theorem 4 and Lemma 1, we can see that system (7) is ZSD and $\operatorname{OFP}\left(-\frac{1}{2}\right)$ and $k_{\delta}\left(\frac{1}{s}+\delta\right)$ is $\operatorname{IFP}\left(k_{\delta} \delta\right)$. Then, the proof follows directly from Theorems 7 and 8. Q.E.D.

Before introducing a numerical example for illustration, we make the following remarks.

\section{Remarks}

- We cannot select a big value of $\delta$ to enlarge the stability margin in practice, because if the fast unmodelled dynamics has a relative degree greater than 1 , this big value of $\delta$ will lead to instability. So, there is a trade-off between robustness and stability.

- The requirement $\delta>\frac{1}{2 k_{\delta}}$ limits the gain $\epsilon$ of the integrator. This is implied by $\epsilon=\frac{1}{\delta}<2 k_{\delta}$. Furthermore, a proper selection of $\epsilon$ can ensure the desired disk margin. 
- If $h(x) \rightarrow 0$ when $k(x) \rightarrow 0$, then the integral controller can suppress a constant input disturbance (see the example in Section VI).

\section{Discussion}

From the previous section, we can see that the key step for the constant disturbance rejection controller design is to design the optimal controller $C_{0}$. Optimal stabilisation guarantees several desirable properties for the closed-loop system, including the stability margin. However, solving the HJB PDE in general is not a feasible task in a direct approach. On the other hand, the robustness achieved as a result of the optimality is largely dependent on a particular choice of functions $l(x)$ and $R(x)$ in the cost function (1). Addressing this difficulty, the inverse optimal design method is introduced in [10]. In this approach, a stabilizing controller is designed first and then shown to be optimal for a cost functional of the form (1). This problem is inverse because the functions $l(x)$ and $R(x)$ are a posterior determined for the stabilizing feedback, rather than a priori chosen by the designer. By using the inverse design, we can achieve optimal stabilization for the cost function (1) without solving the HJ PDE, but when $R(x) \neq I$ the disk margin are generally not ensured. Fortunately, we can achieve a disk margin by using the domination concept (see pp. 103-107 in [10]).

Now, we have a more feasible way to achieve the optimal stabilization for the special case $R(x)=I$ of the cost function (1) and the disk margin is going achieved based on the inverse optimal design and domination.

After having designed the optimal controller $C_{0}$, we augment it with the controller $k_{\delta} \delta\left(1+\frac{\epsilon}{s}\right)$ to suppress the external disturbance. If one denotes $k_{P}=k_{\delta} \delta$ and $k_{I}=k_{\delta} \delta \epsilon$, then the controller $k_{\delta} \delta\left(1+\frac{\epsilon}{s}\right)$ can be regarded as a PI controller.

\section{ILLUSTRATIVE EXAMPLE}

Consider the nonlinear system

$$
\left\{\begin{array}{l}
\dot{x}=x^{2}+u \\
y=h(x)=x^{\frac{2}{3}}+\sin (x) .
\end{array}\right.
$$

For the special case $(R(x)=I)$ of the cost function (1), we can find an optimal stabilizing control as

$$
u^{*}(x)=-k(x)=-x^{2}-x\left(x^{2}+1\right)^{\frac{1}{2}} .
$$

If we select $k_{\delta}=1$ and $\delta=3$, then the closed-loop system shown in Figure 3 should be GAS according to Theorem 9.

We use MATLAB-Simulink to demonstrate the input disturbance rejection effect of the designed integral controller. The block diagram is shown in Figure 4 for cases of the proposed augmented integral-optimal controller and of the optimal control only.

The system output response $y=h(x)$ (with and without integral controller augmentation) is shown in Figure 5. From this figure, we can see that the designed integral controller can totally reject the disturbance effect in the steady state.
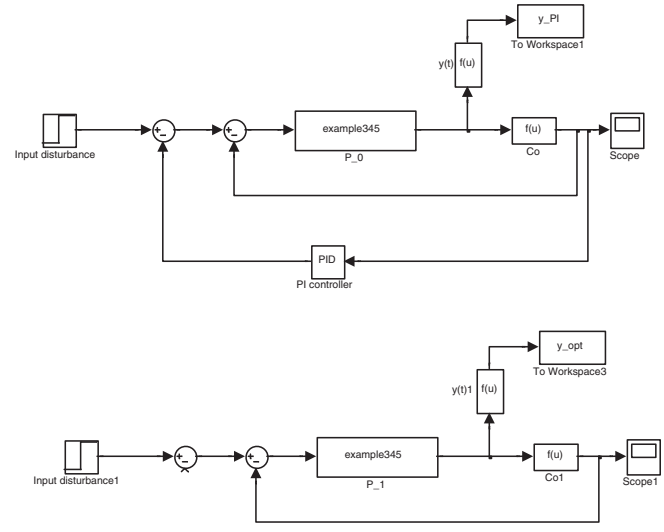

Fig. 4. Simulink block diagram: Proposed integral control (Top), and optimal control only (Bottom).

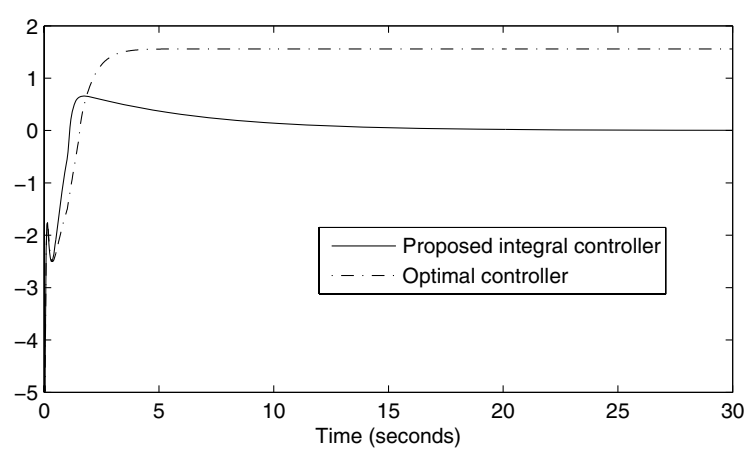

Fig. 5. System output response

\section{CONCLUSION}

In this paper we have presented a nonlinear integral controller design method based on optimal control and passivity theorems. An interesting feature of the proposed method is its capability of suppressing external disturbances in nonlinear systems subject to modelling uncertainties. This is achieved by augmenting the optimal stabilising control with an integral control loop. The design procedure is illustrated by a simple example. Simulation results show that the proposed integral control approach can effectively suppress exogenous disturbance in the steady state.

\section{ACKNOWLEDGMENT}

The third author would like to acknowledge support obtained from the Australian Research Council (ARC) Centre of Excellence for Autonomous Systems.

\section{REFERENCES}

[1] S. Su, B. Anderson, and T. Brinsmead, "Constant disturbance rejection and zero steady state tracking error for nonlinear systems design," in ACCSC'2001, Biswa Datta, Ed. Kluwer, pp. 1-30. 
[2] S. W. Su, B. D. O. Anderson, and T. S. Brinsmead, "Use of integrator in nonlinear $\mathcal{H}_{\infty}$ design for disturbance rejection, submitted," Automatica.

[3] K. Zhou, J. C. Doyle, and K. Glover, Robust and Optimal Control. New Jersey: Prentice-Hall, 1996.

[4] J. W. Helton and M. R. James, Extending $\mathcal{H}_{\infty}$ Control to Nonlinear Systems: Control of Nonlinear Systems to Achieve Performance Objectives. Philadelphia U.A.S.: Society for Industral and Applied Mathematics, 1999.

[5] T. Mita, X. Xin, and B. D. O. Anderson, "Extended $\mathcal{H}_{\infty}$ controlsolving $\mathcal{H}_{\infty}$ servo and estimation problems," Proceedings of the 36th Conference on Decision and Control, pp. 4653-4658, 1997.

[6] T. Mita, M. Hirata, K. Murata, and H. Zhang, " $\mathcal{H}_{\infty}$ control versus disturbance-observer-based control," IEEE Transactions on Industrial Electronics, pp. 488-495, June 1998.

[7] W. C. A. Maas, Nonlinear $\mathcal{H}_{\infty}$ control: the singular case. Netherlands: Centrum voor Wiskunde en Informatica, 1996.

[8] P.Kokotovic, H.K.Khalil, and J.O'Reilly, Singular Perturbation Methods in Control: Analysis and Design. U.S.A.: Academic Press Inc, 1986.

[9] B. Anderson, "The small-gain theorem, the passivity theorem and their equivalence," J. Franklin Inst., vol. 293, no. 2, pp. 105-115, 1972.

[10] R.Sepulchre, M.Jankovic, and P.Kokotovic, Constructive Nonlinear Control. New York: Springer Verlag, 1996.

[11] S. Su, J. Bao, and P. Lee, "Conditions on input disturbance suppression for multivariable nonlinear systems on the basis of feed forward passivity," International Journal of Systems Science, vol. 37, no. 4, pp. 225-233, 2006.

[12] M. Krstic, I. Kanellakopoulos, and P. Kokotovic, Nonlinear and adaptive control design. New York: Wiley Interscience, 1995.

[13] A. J. V. der Schaft, $\mathcal{L}_{2}$ Gain and Passivity Techniques in Nonlinear Control. New York: Springer Verlag, 1996. 